\title{
Simultaneous interpretation training teaching method in college English majors \\ YuGu
}

Zhengzhou university of industrial technology.Henan.China

\begin{abstract}
Keywords: Simultaneous interpretation;English interpretation, college training
\end{abstract}
\begin{abstract}
Simultaneous interpretation as a translation mode with high efficiency and instantaneity is the most common interpretation way in current society. Such translation form can not just effectively improve conference efficiency, but impose no impacts on speakers' thought and contribute to audiences to comprehend the contents of both parties. But such translation mode has very strict requirements for interpreters. This paper conducts in-depth research on interpretation ability training according to features and problems of college English interpretation and explores feasible methods so as to make contributions to Chinese interpretation cause.
\end{abstract}

\section{Introduction}

With the constant acceleration of Chinese reform process, China has increasingly frequent association with various countries in the world in economy, politics and culture and social demands for interpretation talents increase year by year. To improve interpretation ability of college students, colleges and universities respectively explore different interpretation training modes. This paper will analyze and discuss basic quality and working norms of English simultaneous interpreters in detail so as to promote development of Chinese simultaneous interpretation. Though corresponding progress has been made, it cannot meet current social demands and students' needs. Therefore, the improvement of college English interpretation teaching quality and students' interpretation ability and the delivery of professional interpretation talents to the society become the priority among priorities in interpretation teaching of colleges and universities.

\section{Features of translation}

Translation is a field involving a wide range, including written translation and oral interpretation as well as languages of various countries in the world. English written translation mainly refers to translation of newspapers, periodicals and magazines etc. Oral interpretation utilizes oral expression to transform language. But, in oral interpretation process, interpreters need to make sure there is no any delay according to requirements of working norms. According to different occasions, there are different kinds of professional terms and translation tasks. Simultaneous interpretation is the most difficult translation type with the strictest time limit and the highest requirements for language transformation speed and response speed in oral interpretation field. Hence, simultaneous interpretation workers need to own sufficient professional knowledge foundation and excellent flexibility in order to make sure they can adapt this job and offer superior interpretation services for important conferences.

Simultaneous interpretation is the most difficult among all translation forms. Such translation form not just has strict requirements for interpreters' response and language transformation time, and also has high requirement for translation accuracy. Thus, simultaneous interpretation personnel should rapidly listen to, remember, digest, organize, transform and completely express the contents when both parties of a conference begin to speak. In other words, interpreters have to process all contents in their brain immediately and narrate the contents with the most accurate and proper language. So, one can imagine the complexity and difficulty. In the translation process, interpreters should not just digest speech contents, but also correct unofficial and non-normalized language in time. So, if interpreters do not own solid language foundation and flexibility, they cannot complete such work. Simultaneous interpretation is the highest stage of oral interpretation field and even the whole translation field, so the requirements for interpreters are naturally strict. But, simultaneous 
interpretation personnel also enjoy the highest treatment in translation field. Only such preferential treatment can stimulate interpreters to complete translation tasks in each conference with high quality. As China's economic development continuously tends to internationalization, domestic simultaneous interpretation development continuously advances. Especially in economic, business or diplomatic conferences, simultaneous interpretation personnel with solid knowledge base and relevant professional quality must be employed to convey conference contents by virtue of their flexibility and translation skills in order to make sure the conference is completed in a satisfying way and really promote China's economic development. If the qualification of simultaneous interpretation personnel is insufficient, conference content conveying may be not timely, and errors and even conference delay may be caused. Such situation will generate negative effects on the whole conference. If such negative effects happen in large conferences, national relations and economic development will be greatly affected negatively ${ }^{[1]}$.

As early as in late 1970s and early 1990s, some leading universities with iconic significance in Xiamen, Guangzhou and Beijing set up oral interpretation courses in English major and employed professors, scholars and excellent interpretation personnel with rich professional knowledge and oral English education experience for many years to teach simultaneous interpretation knowledge and skills for students. Under the historical background where economic development increasingly tends to internationalization, China's demand for foreign economic activities and international cultural exchange is also boosting. The contact with other countries in economy, culture and politics also becomes closer. Transnational business negotiation is naturally on the rise. Hence, the demand of domestic employment market for simultaneous interpretation personnel is in the rapid increasing stage, and the response is also very hot. Of course, such exuberant demand for talents brings both good opportunities and large challenges to Chinese simultaneous interpretation personnel. Various countries pay more and more attention to selection and cultivation of high-quality simultaneous interpretation talents. So, simultaneous interpretation talents can better adapt requirements of modern society for simultaneous interpretation talents, offer superior services for China's economic and trading activities and smooth development of diplomatic activities and promoting economic stability and sustainable development only through continuously enriching their knowledge and training their flexibility ability.

\section{Basic requirements of simultaneous interpretation Solid bilingual knowledge foundation and excellent flexibility}

Professional simultaneous interpretation personnel should own strong language expression talent and language sensitivity. Besides, they should own the ability to control native language and English. In addition to these, they also should own strong curiosity, exploration desire and strong attention to fresh things, because it is required to concentrate attention and ensure not to be affected so as to more accurately grasp the gist, organize and correct the language and guarantee expression accuracy in simultaneous interpretation process. High concentration is a key emphasis in work and also a difficult point in interpretation process. In view of instantaneity and flexibility features of simultaneous interpretation, the time for thinking and transformation is very limited. Thus, without solid language knowledge foundation and excellent flexibility, simultaneous interpretation personnel cannot transform and express language in time and even will delay conference course. Hence, only comprehensive talents with abundant language knowledge foundation and excellent flexibility who dabble in multi-aspect knowledge can be better qualified for this complex and demanding job ${ }^{[3]}$. As per simultaneous interpretation time requirement in international conference, except language foundation and flexibility, simultaneous interpretation personnel also should own very strong oral expression and linguistic organization ability. The speaking speed should be controlled within 250 words / minute, while general speaking speed is about 100 words / minute. Without professional training, one cannot be qualified for simultaneous interpretation work. Moreover, in speaking process, simultaneous interpretation personnel should ensure authenticity, logic and accuracy of the contents. The words should be expressed clearly and normatively. The intonation should be natural and fluent. 
The contents should be targeted, smooth and clean.

\section{Excellent psychological quality}

The contents of simultaneous interpretation are very extensive, involving economy, politics, culture, science and technology. Simultaneous interpretation is usually selected in large-scale conference. Thus, those predicating in interpretation will be nervous and have errors in speaking due to insufficient psychological quality. Even if they are quite familiar with the content, errors may also occur in the interpretation process due to tension ${ }^{[4]}$. So, good psychological basis of simultaneous interpreters is a major factor influencing interpretation with high efficiency, and strong emotion control ability is also quite necessary. Psychological state control is an effective way to better cope with all kinds of emergencies. Interpreters who can control their psychological states can keep calm in the face of any difficulties and complex situations. This has very great significance for understanding of conference contents and interpretation quality. Thus, simultaneous interpreters also should attach importance to improving their psychological quality while boosting their ability in order to make sure interpretation process proceeds.

Simultaneous interpretation team usually consists of 2-3 members. Each interpreter may interpret for about twenty minutes in each interpretation process, and they take turns to interpret. So, tacit cooperation and harmonious working environment of simultaneous interpretation team members are basic elements to make sure the whole interpretation process proceeds smoothly. Team awareness should be promoted as follows: firstly, the working time bucket and content should be arranged according to advantages of each interpreter so as to make sure advantages of each interpreter can be fully exerted; secondly, preparatory work should be listed as a key link. Detailed communication with interpretation team should be done in advance to give them sufficient preparation time and ensure smooth interpretation process and working efficiency; thirdly, in each interpretation exchange process, interpreters under break should pay attention to the work of team members and do assistant work for interpreters under work. Main contents of assistant work include avoiding external interference and recording conference contents etc.

\section{Working norms of simultaneous interpretation}

Self-adjustment ability is a basic ability that simultaneous interpretation personnel must own and also a core factor of maintaining work quality. To effectively adjust one's state, interpreters need to rationally adjust and organize new language information received, correct errors, amend the omission in order to complete high-quality interpretation. For example, in English, the sentences used to express position and time are usually placed at the end of a sentence as adverbial modifier, the positions of main clause and subordinate clause may be exchanged in the interpretation process. According to specific expression requirements of such sentence pattern, interpreters may predict the content to be expressed. Thus, when hearing I accept your statement, interpreters may complete interpretation.

\section{Development prospect of simultaneous interpretation}

At present, in the whole international market, follow-up development of simultaneous interpretation is optimistic and is regarded as a cause where there is lack of talents and is not short of money. Besides, simultaneous interpreters belong to rare human resources. It is most difficult to cultivate such kind of talents. According to relevant research results, the total number of world-wide professional simultaneous interpreters is about 2000. The demand for Chinese simultaneous interpretation talent resources exceeds supply. Hence, English has popularized at every corner in modern society, but the talents who can become simultaneous interpreters are very scarce. Simultaneous interpreters in minority languages are more rarely seen. As China communicates with various countries in the world frequently and China's international position is on the rise, international conferences that Chinese institutions and enterprises also become more and more. Each 
international conference cannot be held without simultaneous interpreters. Thus, simultaneous interpretation has good development prospect and competition in both domestic market and international market.

\section{Conclusion}

Simultaneous interpretation is a demanding, challenging and long-term occupation and also has many opportunities.Interpretation is a communicative behavior of transforming the information from one language to another in oral form. It is not only theoretical knowledge, but also a skill. Therefore, it can be fully mastered only through a lot of practice and exercise. Students can become qualified interpretation talents only by promoting the spirit of perseverance and paying attention to the use of interpretation methods.Hence, Chinese English talents should enhance English knowledge foundation and professional ability, greatly improve English interpretation skills and enter simultaneous interpretation team in order to drive development of society and economy and achieve the value of English talents.

\section{Reference}

[1] Jiang Renji, On basic quality requirement and basic principle of simultaneous interpretation in English translation. Shenzhou, 2014(8):97-97.

[2] Fang Kemin.Simultaneous interpretation skills and training. Journal of Beijing Polytechnic College, 2004,3(3):72-75.

[3] Zhao Liang. English Listening and Interpretation Brief Discussions on Improvement of Listening Ability in English Interpretation Training. Northern Literature: II, 2012(1):120-121.

[4] Li Mengzhuo. Brief Analysis on Short-term Memory Training for Improving English Interpretation Quality. Kaneda, 2013(9):186.

[5] Lv Sisheng. Test Report on Influence of “Loading Over-speed Interpretation Training Method” on English Interpretation. Hebei Normal University, 2013.

[6] Liu Qingxue. Research on Multi-modal Teaching Model of English Interpretation Course. Leading Edge, 2012(10):160-161. 